\title{
Structure of transformation of the road motion parameters in the control system
}

\author{
Liudmyla Abramova ${ }^{1}$, Yevhen Nahornyi ${ }^{2}$, and Henadii Ptytsia ${ }^{1 *}$ \\ ${ }^{1}$ Kharkiv National Automobile and Highway University, Department of Organization and Road Safety, \\ Kharkiv, Ukraine \\ ${ }^{2}$ Kharkiv National Automobile and Highway University, Department of Transport Technologies, Kharkiv, \\ Ukraine
}

\begin{abstract}
Over the past decades, the world has witnessed an increase in the number of vehicles. According to the accident analysis and to the existing transport problems, the development of automated traffic control systems using adaptive management methods is the most optimal way to improve road traffic quality. From the technological point of view, the system must function according to the requirements for the traffic flow level and to the assessment of traffic efficiency, so a clear comparison of systems is impossible under the conditions of various principles for identifying managerial impacts and the designation of management system. The authors' analysis of the most commonly used traffic management technologies proves that experts choose the system architecture that affects its functions and the ability to implement one or another method of management. In accordance with the ITS approach and management tasks at each hierarchy level, we believe that the traffic management system of the intelligent transport system should be based on the principle of multi-level architecture using ring Ethernet technologies, which corresponds more to the diamond-shaped system structure; it allows to distribute methods of traffic management for purposes and to separate the informational and technological elements of the system.
\end{abstract}

Transport is one of the basic branches of the Ukrainian economy. But the current state of the transport system does not fully meet the requirements for the effective implementation of the European integration course of Ukraine and the integration of the national transport network into the trans-European transport system.

According to the National Transport Strategy of Ukraine for the period up to 2030, it was identified that the creation of competitive and efficient transport system was chosen among the strategy implementation priority directions, according to the world industry trends, namely, the development of intelligent transport systems. In order to implement actions in this direction, common problems were identified: high mortality and injury rate caused by road traffic accidents; imperfection of transport security management systems; low level of introduction and practical application of the latest technologies and technical devices for road traffic organization.

Therefore the development of the intelligent traffic management system foundations is considered appropriate and topical. Over the past decades the world has witnessed a rapid

\footnotetext{
${ }^{*}$ Corresponding author: gennadij.ptitsa@gmail.com
} 
increase of the vehicles quantity and traffic, which leads to an increase of accidents number and their negative consequences. The data determining the urban transport network quality level were obtained with the help of entropy levels as the measure of its parameters inconsistency [1]: St. Petersburg - 3.25; Kyiv - 2.2; Kharkiv - 2.2; Moscow - 1.75; Kaliningrad - 1.6; Riga 1.55; Poznan - 1.3; Vilnius - 1.3; Kaunas - 1.1; Minsk - 1; Warsaw - 0.9; Bratislava - 0.8; Prague - 0.6; Edmonton - 0.5; Helsinki - 0.45, Toronto - 0.4, Berlin - 0.4; Stockholm - 0.2; Oslo - 0.1.

According to the analysis of accidents in the large cities above, the development of automated traffic control systems (ATCS) with the use of adaptive management methods is the most promising and optimal way of the traffic quality improving, as evidenced by the experience of developed countries. At the XXIV Intelligent Transport Systems World Congress held on October 21- November 02, 2017 (Montreal, Canada) it was determined that the ATCS is intended to provide an efficient traffic management in cities with the help of traffic signaling for road traffic safety.

The accumulated world experience in the creation and implementation of ATCS shows that the development of activities in the traffic management systems design allows the following results: reduction of transport delays - 15-40\%; the street-road network capacity increasing $10-15 \%$; reduction of the number of accidents - 15-20\%; and reduction of environmental pollution $-20-25 \%$.

From the technological point of view, ATCS should function according to the requirements of the traffic flow level and the estimation of traffic efficiency, so a clear comparison of the ATCS is not possible under the conditions of various principles for identifying management impacts and designating a management system. The main characteristics of systems are [2]: designation of the ATCS and its zone of operation; architecture (decentralized or centralized); place, method and devices of motor vehicles (MV) monitoring on the city's highway network $(\mathrm{CHN})$; method of control parameters optimizing in the control system; configuration (modularity) of subsystems; selection and definition of road traffic performance criteria (local or systemic); way of regulating traffic.

The authors' analysis [3] of the most commonly used traffic management technologies and well-known traffic control systems such as UTOPIA, SCOOT, SCATS, BALANCE, MOTION, ITACA, ROSE proves that experts choose the architecture of a system that affects its functions and the ability to implement one or another method of management as the main hallmark of management principles. It has been discovered that the hierarchical architecture has the advantage, since it affects the flexibility and mobility of the management system to the coverage area, the configuration of the hardware and subsystems, and also has the ability to respond to the redistribution of traffic efficiency criteria. All this points to the formation of the principles of intelligent traffic management systems, so the adaptive real-time ATCS may not use transport models as a management algorithm and implement digital monitoring methods by means of telecommunications and positioning of online e-mapping (GPS, GPRS).

Well-known studies of intelligent transport systems both in our country and abroad [5] allow us to keep improving the road quality and safety by creating a cyber-physics system for computer online interaction of the driver with cloud services, managing MV on the basis of transport infrastructure digital monitoring and traffic with the use of smart sensors, telecommunication and navigation facilities [6]. However, this innovative approach to the creation of the latest technologies of intelligent traffic control systems, which are aimed at increasing the capacity of the CHN and the time of the MV movement, does not exclude the application of traffic management methods and measures implemented by the ATCS.

At the same time, it should be understood that the transition to each next generation of control systems is associated with significant costs, both for the development and for operation of systems. And in our country at present, in most cases, the existing traffic management systems belong to systems of the first or second generation, where traffic is managed without taking into account the change of transport situation on the CHN. Also speaking about the 
design features of ATCS, it is necessary to attribute conformity of the latest developments to the current standards and political directions of the state.

The design of a traffic management system based on the determination of dynamic object management (traffic) levels requires the formation of the theoretical foundations for the development of information technologies for the intellectual management of distributed control functions by levels based on the development of the structure of the control system parameters transformation.

In the intellectual management system, knowledge about unknown characteristics of the management object and environment are formed in the process of adjustment, and the information obtained in this case is used in the process of automated decision making, which improves the quality of management. At the same time, adjustment can occur in isolation from the management process or directly in the process of automatic control in real time. Integrated intelligent control system is a modification and development of the traffic management system, which implements the technology of situational management of traffic flows in the street-road network of cities.

It should be noted that intellectual and information technologies are to be distinguished. Information technology functions as intellectual support. Intellectual decision-making technologies deserve consideration - they provide an opportunity, along with a solution (or in the course of obtaining a solution) to seek new knowledge and accumulate intellectual resources. Information technology only creates information resources. Thus, knowledge, formalized in explicit form, can be part of the knowledge base in traffic control systems and can be used to make decisions about the choice of driving influences on road traffic.

In most of the existing traffic control algorithms in our country, control influences are aimed at eliminating obstacles to traffic, that is, there is a post-effect effect, because there is no biased management for their prevention. Under the current state of the road, the number of the urban street network sections is constantly increasing, which can be considered as a bottleneck of the transport network and there is a mismatch of traffic parameters with the parameters of the transport network. Consequently, there is a spatial relationship between abnormal and dangerous situations throughout the transport network as a whole, which implies the existence of a mutual relationship between controllers of the network.

One of the most important aspects of the traffic management problem is not only the determination of the totality of management influences and the sequence of their implementation by the management system, which should be distributed with a rational degree of decentralization and function in real time, but the most effective centralized architecture of the system. The indicated differences influence the differentiation in the architecture of the system, the composition of the subsystems for the management levels and the choice of technical support elements, taking into account the fact that intelligent control systems belong to the type of automatic systems.

According to the ITS approach [4], as well as to the management tasks at each level of the hierarchy, we believe that the traffic control system (TCS) of the intelligent transport system should be based on the principle of multilevel architecture using ring Ethernet technologies that more closely corresponds to the diamond-shaped form of TCS structures. It allows to distribute not only methods of traffic control (TC) for purposes, but also to separate informational $\left(i, n^{\text {inf }}\right)$ and technological $\left(i, n^{\mathrm{T}}\right)$ elements of the system (fig. 1). 


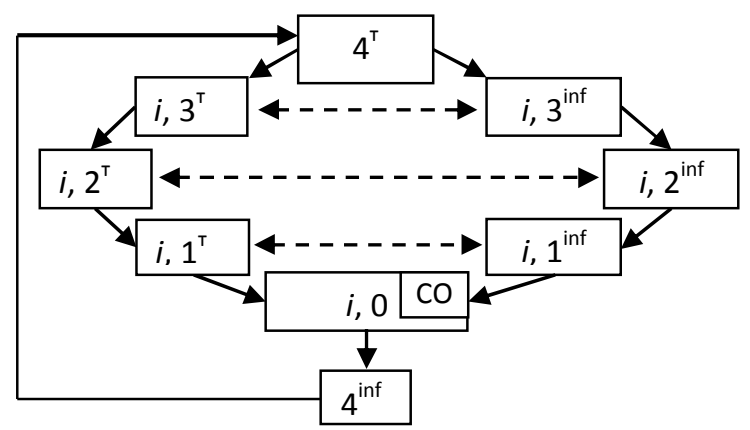

Fig. 1 The structure of the hierarchical traffic control system with two centers.

The term "diamond-shaped structure" of the control system is used to describe the systems under the control of two control centers, namely, information $\left(4^{\mathrm{inf}}\right)$ and technical $\left(4^{\mathrm{T}}\right)$, which are interconnected, we suggest this approach to use in traffic management systems [6]. Each link in a structure with number $i=1, m_{j}$ located on the $j$-th hierarchical level $(j=0, n)$ can be displayed as:

$$
S_{i j}: U_{i j} \times Y_{i j} \times H_{i j} \rightarrow X_{i j}
$$

where $U_{i j}=\left\{u_{i j}\right\}$ - the set of managing influences of a higher level;

$Y_{i j}=\left\{y_{i j}\right\}$ - the set of feedback signals from the lower levels;

$H_{i j}=\left\{h_{i j}\right\}$ - the set of perturbing influences of this link;

$X_{i j}=\left\{x_{i j}\right\}-$ the set of output signals of the link $i$ to the lower level.

For the uppermost part, the expression has the form:

$$
S_{i j}: Y_{m, 4} \times H_{m, 4} \rightarrow X_{4}
$$

And for the control object (CO), a system of mappings is formed as:

$$
S_{m, 0}: X_{i, 1} \times H_{i, 0} \rightarrow X_{0}
$$

But there is a plurality of disturbing influences in expressions (2) and (3), and in TCS we have two types of disturbing influences - external (climatic and weather conditions, the quality of the roadway coverage, the coefficient of adhesion of the wheel with the road, etc.) and internal (change of the traffic flow mode, change of service levels of the transport network). Internal disturbances are of greater importance for the decision-making level, and external disturbances are more important to the center of system management. Therefore, the presence of two vertices in the proposed structure has the basis for the distribution of management functions - managing and decision making, which respectively apply the technological and informational components of the management system. Then each link in the proposed structure can be summed up by two interconnected parts: the first participates in the formation of control influences, taking into account external perturbations $\left(H^{\mathrm{E}} \mathrm{ij}\right)$, and the other influences the assessment of the current state of traffic or performance indicators $\left(H_{i j}^{\mathrm{i}}\right)$. Therefore, the mapping in a diamond-shaped structure for each link will consist of two parts:

- for technical devices

$$
S_{i j}^{\mathrm{T}}: U_{i j}^{\mathrm{T}} \times Y_{i j}^{\mathrm{T}} \times H_{i j}^{\mathrm{E}} \rightarrow X_{i j}
$$

- for the information components of the system

$$
S_{i j}^{\mathrm{inf}}: U_{i j}^{\mathrm{nf}_{i j}} \times H_{i j}^{\mathrm{I}} \rightarrow X_{i j}
$$


Thus, the efficiency of transition to a diamond-shaped structure during the transformation of TCS parameters has advantages in management and allows dividing the control and information channels of the system at all levels of the hierarchy; allocating local dynamic control contours in the system; considering the typical problems of static calculations of control effects taking into account deterministic and fuzzy parameters of traffic; and creating a "convenient" mathematical description of the individual links and the system as a whole.

We propose an approach to the structural formation of management levels. At the first (lower) level the solution of the tasks is provided, based on the principles of automatic regulation (AR), that is, the software changes the parameters of the object in accordance with the settings that are defined and set at the highest tactical level (fig. 2). It includes: control object $(\mathrm{CO})$, technical regulation devices $(\bigotimes)$, local road controller $\left(\mathrm{RC}_{\mathrm{L}}\right)$ and control algorithm $(\mathrm{CA})$ for traffic lights $\left(\mathrm{T}_{\mathrm{C}}\right)$.

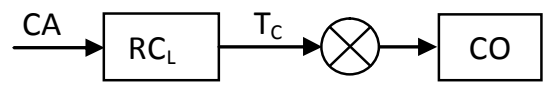

Fig. 2 The structural scheme of automatic regulation.

The principle of an AR is "rough" (robust) to change its input parameters. Robust management is a set of methods of the management theory, whose purpose is to determine the controlling influence in the presence of not complete and not detailed description of the management object. A special place among the concepts of the robust control theory is the uncertainty of the input parameters; it reflects the inaccuracy of control parameters. The work involves a small and timely change in the output of the control system open loop $\left(\mathrm{T}_{\mathrm{C}}\right)$ when the parameters of the control object, such as intensity $(N)$, composition $(C)$ and speed $(V)$ of the traffic flow, are changed. This is a main disadvantage of this kind of control.

This disadvantage can be avoided with the help of adaptive management. Thus, the management processes for objects whose parameters are unknown from the beginning of the system's operation are formed, and the property of adaptation is achieved by forming a mathematical model of the control object to determine the impact on it. Therefore, the peculiarity of adaptive control systems (ACS) is the possibility of obtaining input information for the formation of control impacts and the availability of a priori information regarding the uncertainty in the system. This is a fundamental distinction between adaptive control and robustness at the second tactical level of traffic control (fig. 3); they include transport detectors (TD) and road system controllers $\left(\mathrm{RC}_{\mathrm{S}}\right)$ forming control influences $u(t)$.

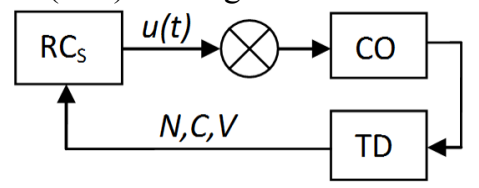

Fig. 3 The structural scheme of adaptive control systems.

The ACS level is designed to manage a limited number of objects subordinated to the corresponding local control units. Criteria for the purpose of management, acting on this level, may differ from the general criterion of the whole system functioning.

At the third strategic level, coordination and optimization of management are being implemented to coordinate the work of local lower level control devices to achieve the overall goal of the operation of the entire management system as a whole. Determining the optimal driving influence on traffic in urban conditions is a complex task with a large dimensionality of parameters. The mutual influence of the control object parameters, the technical means of regulation and the street-road network (parameters of lower levels of management) forms the conditions and limitations to the mathematical apparatus in the development of the management algorithm and decision-making process, in relation to the choice of control influence, carried out at the highest level of management. 
The level of operational management and decision-making includes, in addition to the TCS technical support center, a governing body (a team of experts or a decision maker), equipped with modern computer technology to computer possible solutions and choose the most effective option for resource allocation management between individual subsystems and decision making in various emergency situations. The ideal variant to ensure the effective operation of the TCS is to use "fast models" to lower levels of the hierarchy and control object using the elements of the information control system (the formation of bases of control models, databases) for the operational level of management. This approach stipulates the requirements for the design of a hybrid traffic management system based on the received structural and functional description of the methods distribution and algorithms for traffic management.

Thus, the proposed approach to the distribution of management functions on the basis of the developed diamond-like structure gives the system the ability to clearly distinguish and link the task of two types of analysis and synthesis of dynamic control circuits: which can be structured in the course of "cross sections" of the vertical structure and the tasks of static calculation, and that can be formalized when performing horizontal "cuts" of the structure at each of the levels of the hierarchy of the information or control part of the system. In the conditions of incomplete, fuzzy and inaccurate knowledge of the characteristics of the street-road network, parameters of the traffic flow and environmental characteristics, the use of adaptive control methods is a rather promising direction for the construction of the TCS in the real-time mode. These methods are based on the search for expert knowledge of management and the use of fuzzy simulation, where the approximate model of the object is configured using simulation models that describe the possible states of the system, or the use of self-tuning control using hybrid systems.

\section{References}

1. D.V. Kapskiy, D.V. Navoy. Development of the automated road traffic control systems in Minsk as part of the intellectual city transport system. Science \& Technique. 16 (1), (2017) 38 (in Russian) Available at: https://doi.org/10.21122/2227-1031-2017-16-1-38-48

2. Traffic Control Systems Handbook, U.S. Department of Transportation Federal Highway Administration, available at: https:/ops.fhwa.dot.gov/publications/ fhwahop06006/index.htm

3. L.S. Abramova, Y.V. Nahornyi, CONCEPTUAL APPROACH TO THE TRAFFIC CONTROL SYSTEMS DESIGN. Avtomobil' i elektronika. Suchasni tehnolohiyi [Car and electronics. Modern tehnology]. 12, (2017) 94 (in Ukrainian) Available at: http://oldwww.khadi.kharkov.ua/fileadmin/P_SIS/AE17_2/index.html

4. V. Hahanov, W. Gharibi, B. A. A. Abbas, S. Chumachenko, O. Guz, E. Litvinova. Cloud traffic monitoring and control, Proceedings of the IEEE 7th International Conference on Intelligent Data Acquisition and Advanced Computing Systems (IDAACS), Berlin, Germany, 244 (2013) Available at: https://ieeexplore.ieee.org/ abstract/document/6662681

5. A. Ziarmand, D. Kuchenenko, T. Soklakova Transport monitoring and control systems, Proceedings of the IEEE East-West Design \& Test Symposium (EWDTS), Yerevan, Armenia, 474 (2016) Available at: https://ieeexplore.ieee.org/document/7807662

6. L.V. Derbunovich, L.S. Abramova. Hierarchical structures of traffic management systems, Visny'k NTU KPI, Avtomatyka ta pryladobuduvannya [Bulletin of the National Technical University KhPI, Automation and equipment]. 31, (2008) 40 (in Russian) Available at: https://archive.kpi.kharkov.ua/View/28226/ 